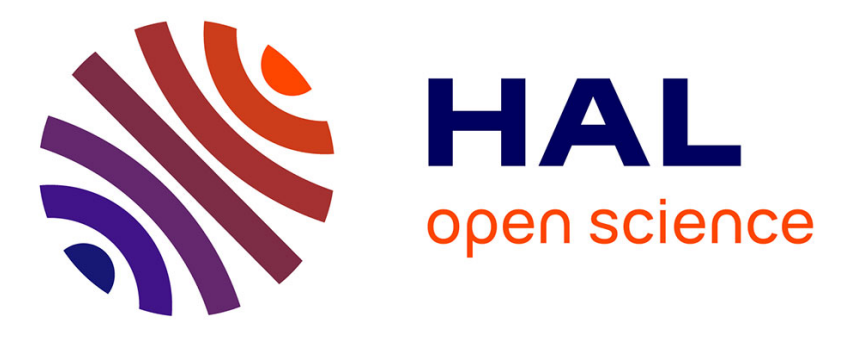

\title{
Integrating safety distances with trajectory planning by modifying the occupancy grid for autonomous vehicle navigation
}

Hafida Mouhagir, Reine Talj, Véronique Cherfaoui, François Aioun, Franck

Guillemard

\section{To cite this version:}

Hafida Mouhagir, Reine Talj, Véronique Cherfaoui, François Aioun, Franck Guillemard. Integrating safety distances with trajectory planning by modifying the occupancy grid for autonomous vehicle navigation. 19th IEEE International Conference on Intelligent Transportation Systems Conference (ITSC 2016), Nov 2016, Rio de Janeiro, Brazil. pp.1114-1119. hal-01378952

\section{HAL Id: hal-01378952 \\ https://hal.science/hal-01378952}

Submitted on 12 Oct 2016

HAL is a multi-disciplinary open access archive for the deposit and dissemination of scientific research documents, whether they are published or not. The documents may come from teaching and research institutions in France or abroad, or from public or private research centers.
L'archive ouverte pluridisciplinaire HAL, est destinée au dépôt et à la diffusion de documents scientifiques de niveau recherche, publiés ou non, émanant des établissements d'enseignement et de recherche français ou étrangers, des laboratoires publics ou privés. 


\title{
Integrating safety distances with trajectory planning by modifying the occupancy grid for autonomous vehicle navigation
}

\author{
Hafida Mouhagir ${ }^{1,2}$, Reine Talj ${ }^{1}$, Véronique Cherfaoui ${ }^{1}$, François Aioun $^{2}$, Franck Guillemard ${ }^{2}$
}

\begin{abstract}
The goal of the work in this paper is to use occupancy grid in integrating safety distances with the planning strategy for autonomous vehicle navigation. The challenge is to avoid static and dynamic obstacles at high speed with respect to some specific road rules while following a global reference trajectory. Our local trajectory planning algorithm is based on the method of clothoid tentacles. It consists on generating clothoid tentacles in the egocentered reference frame related to the vehicle. Using information provided from sensors, we build an occupancy grid that we modify to take into consideration safety distances. We use this modified occupancy grid to classify each tentacle as navigable or not navigable. By formulating the problem as Markov Decision Process, only one tentacle among the navigable ones is chosen as the vehicle local reference trajectory.
\end{abstract}

\section{INTRODUCTION}

Autonomous vehicle (AV) technologies have the potential to significantly improve transportation safety and offer immense social, economic and environmental benefits. As driving functions become increasingly automated, not only do technical specifications and safety regulations become increasingly outdated, but there is also a shift in responsibility from the human driver to the vehicle itself. Thus recently, there have been many studies aimed at increasing the safety of autonomous vehicles [1][2].

One of the most important qualities expected from an AV is avoiding collision with static and dynamic obstacles [3] in its path beside road following, lane keeping and lane change. There are different theoretical and experimental works for road following, lane keeping [4] [5] and lane changing [6][7] [8] using geometric reasoning, control theory or other methods. Some of them impose specific constrains on the dynamic variables of the vehicle and some specifically minimize parameters like time, distance, acceleration, curvature and such.

In [9] and [10], the authors analyzed the obstacle avoidance problem through planning techniques. They used RRT algorithm (Rapidly exploring Random Trees) and lattice planners. Both techniques sample the state space using data structures (trees and lattices respectively), trying to explore it in a quick and safe fashion. Quick exploration is accomplished in both cases and a series of possible paths are provided to

The authors are with ${ }^{1}$ Sorbonne universités, Université de Technologie de Compiègne (UTC), CNRS Heudiasyc UMR 7253, ${ }^{2} P S A$ Groupe, Direction scientifique, Centre technique de Vélizy, France. Email: \{hafida.mouhagir, reine.talj, veronique.cherfaoui\}@hds.utc.fr, \{franck.guillemard, françois.aioun\}@mpsa.com the planning module for the vehicle to follow. However, the planning horizon is claimed to be relatively large and, with regard to the dynamic nature of on-road driving, where obstacles or obstructions appear suddenly, re-planning routines are needed to supplement these incremental search approaches. Therefore, some of the approaches use a limited horizon, both in terms of time and space. One of these approaches is the tentacles method.

The tentacles method uses a set of virtual antennas that are called tentacles and an egocentric occupancy grid around the vehicle [11]. The occupancy grid expresses the state of the environment surrounding the vehicle and contains the obstacles if they exist. The tentacle is a geometric shape that models a possible trajectory of the vehicle and we can find in the literature multiple shapes of tentacles. In [11], the shape adopted for tentacles is circular. The weakness of this approach appears in considering all the tentacles generated for a certain speed as trajectory candidates even if their curvature is not well-suited to the current vehicle steering angle. An improvement has been made by introducing clothoid tentacles method [12]. The clothoid approach considers the current dynamical state of the vehicle and makes a smooth variations in the vehicle dynamic variables. Other notable works are summarized in the survey [13].

This paper is based on an original method for trajectory planning with clothoid tentacle, to avoid static obstacles and follow the reference trajectory, coupled with a decision process inspired from well known MDP (Markovian Decision Process) model, this previous work is described in [14]. This work is distinguished by three key contributions. The first is replacing static obstacles by dynamic ones. The second is the introduction of lateral and longitudinal safety distance while overtaking. The third contribution is the validation of the algorithm with higher speed with respect to the real road dimensions.

The paper is organized as follows: Section II presents our navigation strategy based on the method of clothoid tentacles in a occupancy grid and Markov Decision Process. Section III explains how we integrate longitudinal and lateral safety distance by using occupancy grid. The simulation results based on data taken from $\mathrm{SCANeR}^{\mathrm{TM}}$ studio simulator [15] are discussed in Section IV. Finally, conclusions and perspectives are given is Section $\mathrm{V}$. 


\section{Presentation OF THE NAVIGATion StRAtegy}

The trajectory planning goal is the computation of an obstacle free route from an initial position to a final position while following a desired global reference trajectory defined on a global map. The vehicle must be able to interact coherently with its surrounding environment by using perception information and by efficiently utilizing these descriptions in a short-term planning and decision making. Our trajectory planning strategy is divided into three main steps (Fig. 1):

- Creating and updating occupancy grid with data coming from exteroceptive sensors.

- Generating tentacles which will represent dynamically feasible trajectories.

- Choosing the best tentacle that the vehicle will execute.

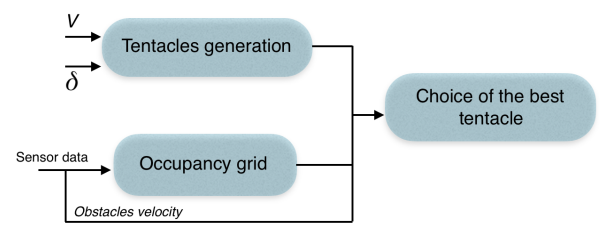

Figure 1: Trajectory planning strategy $(V$ is the vehicle velocity and $\delta$ is the current steering angle)

\section{A. Generating clothoid tentacles}

At a local on-road level, one of the most popular technique of trajectory planning is based on a search space which contains a certain geometric curve (e.g. clothoids or splines) and several lateral shifts of this curve. The method of tentacles consists of using a set of virtual antennas called tentacles in the egocentered reference frame related to the vehicle. Tentacles are a geometrical shape which models the dynamically feasible trajectories of the vehicle. Several forms of tentacles exist: circular tentacles [11] and clothoid tentacles [12][16]. In our work, we use clothoid tentacles because this method considers the current dynamical state of the vehicle and make smooth variations in the vehicle dynamic variables such as the yaw rate, the sideslip angle and the steering angle.

Clothoid is a curve whose curvature varies linearly with curvilinear abscissa, also known as an Euler spiral, Cornu spiral or linarc. Its expression is presented by (1):

$$
\rho=\frac{2}{k^{2}} s
$$

where $\rho$ is the clothoid curvature, $s$ is the curvilinear abscissa and $k$ is a constant, representing the clothoid parameter.

For a fixed speed, all the tentacles begin at the center of gravity of the vehicle and take the shape of clothoid. Every clothoid represents a trajectory with a specific steering angle. Tentacles with the largest bent (those of the extremity) correspond respectively to the positive and negative maximal value of the steering angle which the vehicle can make at the current speed without losing stability. The length of tentacles increases with the increase of the speed.

We assume that all tentacles generated for a given speed $V_{x}$ have the same length:

$$
L_{\text {tentacle }}(m)=\left\{\begin{array}{cc}
t_{0} V_{x}-L_{0} & V_{x}>1(\mathrm{~m} / \mathrm{s}) \\
2(m) & V_{x} \leq 1(\mathrm{~m} / \mathrm{s})
\end{array}\right.
$$

where $t_{0}=7 \mathrm{~s}$ and $L_{0}=5 \mathrm{~m}$.

The initial curvature $\rho_{0}$ of the tentacles is calculated from the current vehicle steering angle $\delta_{0}$.

$$
\rho_{0}=\frac{\tan \delta_{0}}{L}
$$

where $L$ is the vehicle's wheelbase. The set of calculated clothoids depends on the vehicle speed and corresponds to feasible trajectories of the vehicle without losing stability till some defined distance (Fig. 2).
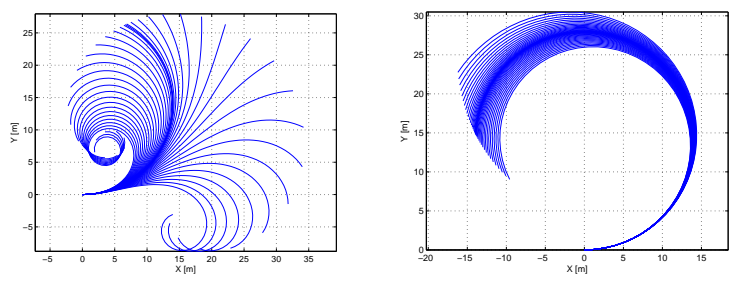

(a) $V_{x}=6 \mathrm{~m} / \mathrm{s}, \delta_{0}=0.3 \mathrm{rad}$

(b) $V_{x}=10 \mathrm{~m} / \mathrm{s}, \delta_{0}=0.3 \mathrm{rad}$

Figure 2: Examples of set of clothoid tentacles

After generating the tentacles in order to guarantee a secure navigation, a security region is generated around each tentacle (see state definition in II-C for details). This area takes into account the width of the vehicle plus a security margin. Using the superposition of the security area within a security distance with the occupancy grid, the navigable tentacles are obtained. The final step of the method is to choose the best tentacle from several navigable tentacles. The best tentacle is then considered as a trajectory to execute for a time sampling duration. If there is no navigable tentacle, the algorithm chooses the tentacle having the greatest distance to the first obstacle and they proceed to brake the vehicle with a constant deceleration along this tentacle.

In order to choose the best tentacle, we use a method inspired from the Markov Decision Process.

\section{B. Occupancy grid}

The occupancy grid representation employs a multidimensional (typically 2D or 3D) tessellation of space into cells, where each cell stores a probabilistic estimate of its occupancy state. The occupancy grid is built by a mapping process that integrate sensor data coming from exteroceptive sensor (Camera, Lidar, Radar) and the pose of the vehicle.

A 2D egocentered grid is constructed as a discrete representation of the environment around the vehicle by a set 
of square cells. Each cell contains information about the occupancy of the corresponding surface. There are various frameworks used for creating and updating occupancy grid like Bayesian framework and evidential framework. Fig. 3 shows an occupancy grid [17] example based on evidential theory.

For evidential theory (also called Dempster-Shafer theory or Belief function theory), the mass is assigned to all subset of the domain which able this theory to represent uncertainty and conflict. In Fig.3, the green color in the occupancy grid shows the navigable space, the red shows the occupied space, while the blue represents conflicting cells and the black represents unexplored cells. The color intensity reflects the certainty degree. In this paper, only binary grids are considered (free/occupied cells)( Fig. 3).
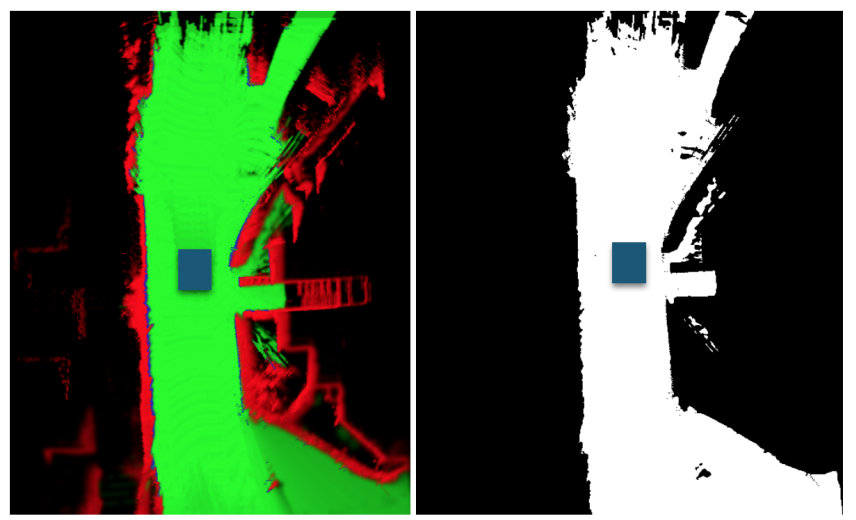

Figure 3: Occupancy grid example. The rectangle represents the vehicle

\section{Proposed MDP approach for trajectory planning with tentacles}

A Markov Decision Process (MDP) is a discrete-time state-transition system. It offers a framework for autonomous robot navigation in dynamic environments [18]. It can be characterized by a finite set of states $S$, a set of actions $A$, and a reward function $R$, transition probabilities $T$, and discount factor $\gamma$. The actions are characterized by transition probabilities distributions, and we write $T\left(s^{\prime} / s, a\right)=p$ to denote that $s^{\prime}$ is reached with probability $p$ when action $a$ is performed in state $s$.

$$
\forall s \in S \forall a \in A \sum_{s^{\prime} \in S} T\left(s^{\prime} / s, a\right)=1
$$

The agent always knows what state it is in. A real-valued reward function $R: S \times A \rightarrow \mathbb{R}$ reflects the objectives, tasks and goals to be accomplished by the agent, with $R(s, a)$ denoting the utility of being in state $s$ and taking action $a$. Discount factor $\gamma \subset[0,1)$ is the discount rate used to calculate the long-term attenuation.

At each of a sequence of discrete time steps (Fig. 4), the agent perceives the state of the environment, $s_{t}$, and selects an

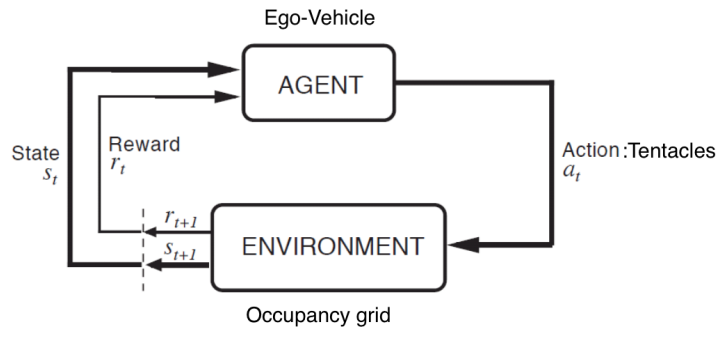

Figure 4: The MDP principle

action, $a_{t}$. In response to the action, the environment changes to a new state, $s_{t+1}$ and emits a scalar reward, $r_{t+1} \in \mathbb{R}$ The agent's goal is to maximize the total amount of reward it receives over the long run. More formally, in the simplest case, the agent should choose each action $a_{t}$ so as to maximize the expected discounted return over the planning horizon $h$ (which could be infinite). This is usually referred to as the value $(V)$ :

$$
V=\sum_{t=0}^{h} \gamma^{t} r_{t}
$$

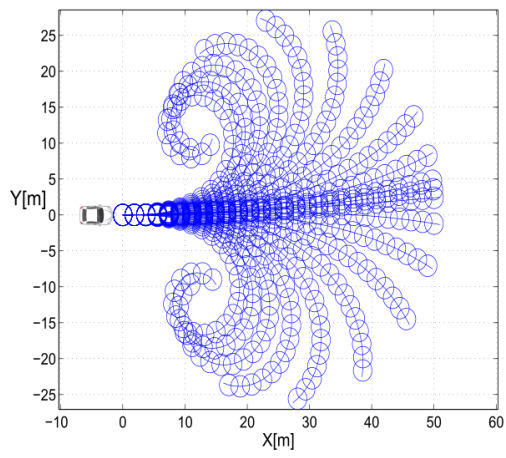

Figure 5: States of MDP model with clothoid tentacles

As described previously, we have to define the states of the system, the actions that can be taken, the transition matrix and the reward that the agent will receive for each action.

- States: are represented by circles $s_{i}$ around the tentacles (Fig. 5), their diameter represent the width of the vehicle with a margin of security. Each tentacle is composed of $n_{s}$ states, and we dispose of $n_{t}$ tentacles. After assigning for every state a reward regarding if it's free, occupied and close from the reference trajectory, we merge the states $s_{i}$ of every tentacle in order to have just one state $s_{f}$ per tentacle.

- Actions: we dispose of $n_{t}$ actions because each tentacle represents an action.

- Transition probabilities: in this work, we assume that we don't have a possible transition from one tentacle to another. Thus we choose deterministic transitions; for all states $s$ and all $a \in A(s)$ we assume that $p\left(s^{\prime} \mid s, a\right)=1$ for a unique $s^{\prime} \in S$, while $p\left(s^{\prime \prime} \mid s, a\right)=0$ for all $s^{\prime \prime} \neq s^{\prime}$ 
. Thus each action leads deterministically from one state to another (or the same).

- Reward: we will define a different reward for every state depending on its occupancy degree $\left(R\left(s_{i \mid \text { occupied }}\right)=R_{O}\right.$ and $\left.R\left(s_{i \mid \text { free }}\right)=R_{f}\right)$, and its closeness to the reference trajectory $\left(R\left(s_{i \mid \text { trajectory }}\right)\right)$. Moreover, we add a positive reward if the tentacle is one of the left side tentacles $\left(R\left(s_{f} \mid l e f t\right)\right)$ because the overtaking is always done on the left side of the vehicle that precede us, in order to avoid ambiguity in case of total symmetry of tentacles $\left(\delta_{0}=0\right)$ when executing an overtaking manoeuvre.

Using occupancy grid, rewards are defined as following:

$$
\begin{aligned}
R\left(s_{f}\right)= & \sum_{k=0}^{n_{s}} \gamma_{t}^{k} R\left(s_{i_{k} \mid \text { trajectory }}\right)+\sum_{k=0}^{n_{s}} \gamma_{o}^{k} R\left(s_{i_{k} \mid \text { occupied }}\right) \\
& +\sum_{k=0}^{n_{s}} \gamma_{f}^{k} R\left(s_{i_{k} \mid \text { free }}\right)+R\left(s_{f} \mid \text { left }\right)
\end{aligned}
$$

where $\gamma_{t}, \gamma_{o}$ and $\gamma_{f}$ are discount factors that can be used to change the behaviour of our approach, and that represent distance attenuation of each kind of reward.

Details regarding the choice and the calculations of each parameters is given in [14]. However, some changes were made, to our original approach. In our previous paper, the tentacle is classified as non-navigable if an obstacle is detected within a distance less than the collision distance $l_{c}$ which is the distance needed to stop a vehicle traveling with a speed $V_{x}$ , with a maximum longitudinal deceleration $a_{m}=1.5 \mathrm{~m} / \mathrm{s}^{2}$ that maintains passenger comfort. It is calculated by:

$$
l_{c}=\frac{V_{x}^{2}}{2 a_{m}}+l_{s}
$$

Where $l_{s}$ is a security marge. If the obstacle is beyond $L_{o}$ the tentacle is classified as navigable. At higher speed, the collision distance increases and knowing that the road borders are considered as obstacle, it becomes harder to find a navigable tentacle along this distance. Thus, we replaced the collision distance by a safety distance which corresponds to the distance travelled during one second, with a given speed. We changed also the expression of $R\left(s_{i \mid \text { trajectory }}\right)$ (eq. 6) in order to make the reference trajectory more attractive for the ego-vehicle:

$$
R\left(s_{\mid \text {trajectory }}\right)=10^{4} / d
$$

In our previous work, we validate the algorithm by testing an overtaking scenario with static obstacle. The next step is to validate it with dynamic obstacle and to respect traffic rules. In the next section, we explain how we integrate traffic rules using the occupancy grid.

\section{INTEGRATION SAFETY DIMENSIONS USING THE OCCUPANCY GRID}

In order to respect safety distances, we modify the occupancy grid (Fig. 8). We suppose that we dispose of sensor that provide information about the position and the velocity of each obstacle.

Suppose an autonomous vehicle is driving at a velocity $V_{1}$. In front of it another car (obstacle) is driving at a constant velocity $0<V_{2}<V_{1}$ in the same direction. The ego-vehicle intends to overtake it.

According to highway code: for a safe driving during an overtaking manoeuver, two conditions must be respected $V_{1}-$ $V_{2} \geq 20 \mathrm{~km} / \mathrm{h}$ and the vehicle must leave a safe lateral and longitudinal distance with other vehicles.

- Lateral distance: according to traffic rules in France, when passing a pedestrian or two wheeled vehicle, the vehicle must leave $1 \mathrm{~m}$ space in urban area and $1.5 \mathrm{~m}$ outside urban area. Inspired by this rule and since regular road width for a freeway is $3.5 \mathrm{~m}$ and the regular vehicle width is $2 \mathrm{~m}$ [19] (Fig. 6). If we suppose that during an overtaking manoeuvre the ego-vehicle passes through the middle of the other lane, then the lateral distance that it must keep with other vehicles is about $1.5 \mathrm{~m}$.

- Longitudinal distance: according to traffic rules, the vehicle must keep 2 seconds of reaction time during ideal conditions. The distance travelled during those 2 seconds is called longitudinal safety distance (Fig. 7).

When overtaking, the vehicle must leave a safe longitudinal and lateral distance with the vehicle it's overtaking; the longitudinal distance as described below is the distance over 2 seconds with the ego-vehicle speed $\left(S D_{1}=2 s * V_{1}\right)$; and since the ego-vehicle must not return to the line of traffic until it's far enough past the other vehicle to avoid a collision, then it must leave a second safety distance with the vehicle behind it, which depends on this other vehicle's speed $\left(S D_{2}=2 s * V_{2}\right)$.

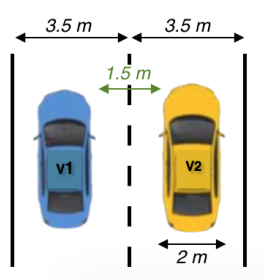

Figure 6: The road width and lateral safety distance

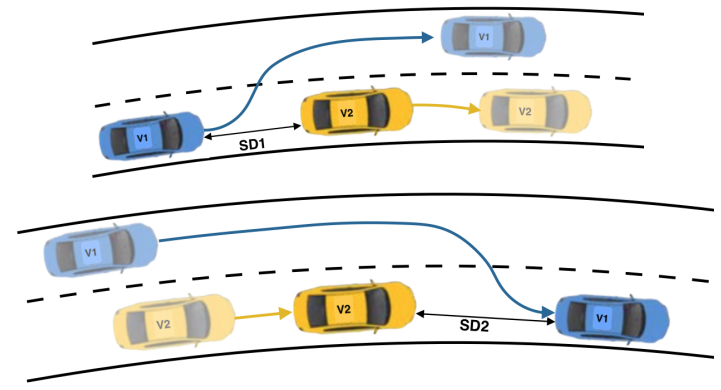

Figure 7: Longitudinal safety distance 
In order to respect safety distances, we expand laterally and longitudinally the obstacle in the occupancy grid (Fig. 9). The actual dimensions of the obstacle are represented by a circle of diameter $2 \mathrm{~m}$, we expand its beam by $0.5 \mathrm{~m}$ from each side in order to respect lateral distance. As it was mentioned in II-C, the tentacle is classified as navigable if no obstacle is detected within a distance less than the distance travelled during one second with a given speed which is in our case $S D_{1} / 2$ Then to satisfy the safety distance over 2 seconds, we need to expand the obstacle width by $S D_{1} / 2$ in the back and by $S D_{2}$ in the front.

The expansion is made by adding circles with varying diameter which satisfies the following equation:

$$
\begin{gathered}
d_{i-\text { front }}=d_{0}-i \frac{d_{0}-0.5}{S D_{2}} \quad i=1 . . S D_{2} \\
d_{i-\text { back }}=d_{0}-i \frac{d_{0}-0.5}{S D_{1} / 2} \quad i=1 . . S D_{1} / 2
\end{gathered}
$$

with $d_{0}=3 m$ is the diameter of the circle representing the vehicle.

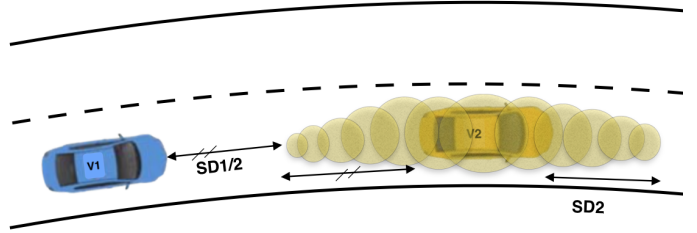

Figure 8: The obstacle shadow after a lateral and longitudinal expansion

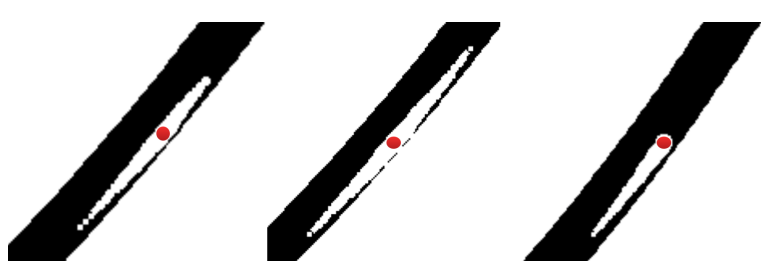

Figure 9: A view of the obstacle expansion in the grid with $V_{1}=20 \mathrm{~m} / \mathrm{s}$ and $V_{2}=5 \mathrm{~m} / \mathrm{s}$ in the left, $V_{2}=10 \mathrm{~m} / \mathrm{s}$ in the middle and $V_{2}=0 \mathrm{~m} / \mathrm{s}$ in the right

\section{Simulation Results}

\section{A. System set-up}

In this part we report the simulation results based on data taken from SCANeR ${ }^{\mathrm{TM}}$ studio simulator. The data taken from the SCANeR ${ }^{\mathrm{TM}}$ studio was processed and simulated in Matlab. With these data, we generate a global map indicating the reference trajectory with its right and left borders and obstacles with red circles (Fig. 10). We indicated the navigable space of the road by black cells having the value " 0 " and the nonnavigable space by white cells having the value " 1 ".
According to the traffic rules, it is necessary to have a sufficient field of view around the ego-vehicle in order to decide if the overtaking is safe or not. This field of view increases with higher speed. Considering the limited sensing range of the actual perception sensors and without communication between vehicles, an overtaking manoeuvre can't be done safely in two sense traffic road. Thus, in our work, we use the highway which is composed of two-lane road with a single sense of traffic.

At every sampling period (100 ms in our case) the sensor provides a new measure and the occupancy grid, considered to be ego-centered around the vehicle, is built. This 2D occupancy grid is constituted of $800 * 800$ cells. The size of each cell is $25 \mathrm{~cm} * 25 \mathrm{~cm}$. We suppose that the frequency used for grid generation is sufficient to ensure safe navigation.

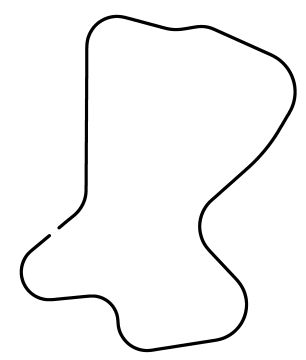

Figure 10: Global map of a test scenario from SCANeR ${ }^{\mathrm{TM}^{\mathrm{S}}}$ studio simulator

The parameters of the MDP like-model are provided in [14]. We changed the number of circles per tentacles $\left(n_{s}=50\right)$. Since we use higher speed, the tentacle's length increases. Then we needed to cover more space.

\section{B. Results}

We evaluate the presented approach with a scenario of overtaking manoeuvre of a dynamic obstacle. We generate the local map and apply our algorithm to choose the tentacle to follow at each sampling step. The validation was done with different speed for the ego-vehicle: $10 \mathrm{~m} / \mathrm{s}$ and $20 \mathrm{~m} / \mathrm{s}$, and for the vehicle in front: $10 \mathrm{~m} / \mathrm{s}$ and $5 \mathrm{~m} / \mathrm{s}$.

In Figures 11 and 12 present the reactions of the algorithm when the ego-vehicle overtake an obstacle (speed: $10 \mathrm{~m} / \mathrm{s}$, $5 \mathrm{~m} / \mathrm{s}$ ) with $20 \mathrm{~m} / \mathrm{s}$ and $10 \mathrm{~m} / \mathrm{s}$ speed. The red line represents the reference trajectory, the blue: trajectory of the ego-vehicle trajectory, the yellow: the middle of the left lane, the black: the borders of the road. The first star is the obstacle position at the beginning of the overtaking and the second one is the position of the obstacle at the end of the overtaking. We observe that the vehicle can make an overtaking manoeuvre with respect to lateral and longitudinal safety distances.

The results ( Table. I )show that our approach respect the lateral and longitudinal safety distances. We can characterize our algorithm by being careful when returning to the reference trajectory. The use of clothoid tentacle includes already 


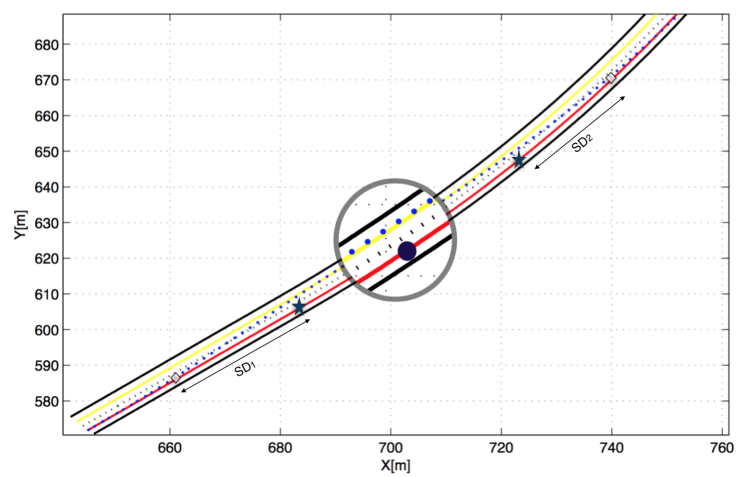

Figure 11: Overtaking manoeuvre and following the reference trajectory with $V_{1}=20 \mathrm{~m} / \mathrm{s}$ and $V_{2}=10 \mathrm{~m} / \mathrm{s}$.

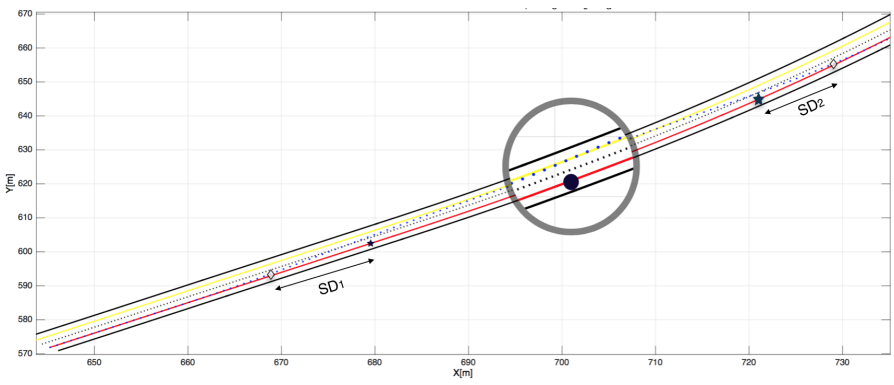

Figure 12: Overtaking manoeuvre and following the reference trajectory with $V_{1}=10 \mathrm{~m} / \mathrm{s}$ and $V_{2}=5 \mathrm{~m} / \mathrm{s}$

a safety distance. This form of tentacles makes a smooth variations in the vehicle dynamic variables such as the yaw rate, the sideslip angle and the steering angle. Thus the return to reference trajectory is not sharp. We work in reducing $S D_{2}$ by making the reference trajectory more attractive and quantifying the safety distance integrated into the tentacle approach.

\section{Conclusion And Perspectives}

In this work, the goal is to integrate considerations of respecting the traffic rules in the planning algorithm. The simulation results show good performance of our algorithm in avoiding dynamic obstacles with respect to safety distances. In fact, this reactive algorithm does not accumulate data and it takes into account vehicle dynamics and real road structure by using the shape of the clothoid. Among the perspectives, we aim to consider the uncertainty of the environment surrounding the vehicle. This improvement would be at the perception level, by using an occupancy grid based on evidential theory, that can be considered in the reward calculation. Further, an interesting extension of our method would be to consider the uncertainty on actions by introducing stochastic transitions instead of deterministic ones.

We also look to implement our algorithm in a robotized vehicle in order to demonstrate the validity of the algorithm.

\begin{tabular}{|c|c|c|c|c|c|c|}
\hline$V_{1}$ & $V_{2}$ & $S D_{1}$ & theo. $S D_{1}^{\prime}$ & $S D_{2}$ & theo. $S D_{2}^{\prime}$ & $L D$ \\
\hline \hline 20 & 10 & 38.7 & 40 & 27 & 20 & 1.5 \\
\hline 20 & 5 & 39 & 40 & 15 & 10 & 1.4 \\
\hline 10 & 5 & 20.5 & 20 & 16 & 10 & 1.5 \\
\hline
\end{tabular}

Table I: Approximate measurements of longitudinal and lateral distances. Velocity $(\mathrm{m} / \mathrm{s})$ and distance $(\mathrm{m}) . S D_{1}^{\prime}$ and $S D_{2}^{\prime}$ represent the theoretical distances to respect.

\section{REFERENCES}

[1] U. Ozguner, C. Stiller, and K. Redmill. Systems for safety and autonomous behavior in cars: The darpa grand challenge experience. PROCEEDINGS-IEEE, vol. 95, no 2, pp. 397, 2007.

[2] A. Rizaldi and M. Althoff. Formalising traffic rules for accountability of autonomous vehicles. International IEEE Conference on Intelligent Transportation Systems, 2015.

[3] J. Hardy and M. Campbell. Contingency planning over probabilistic obstacle predictions for autonomous road vehicles. IEEE Transactions on Robotics, pp. 913-929, 2013.

[4] J. Ackermann and W. Sienel. Robust control for automatic steering. In American Control Conference IEEE, pp. 795-800, 1990.

[5] M. A. Sotelo. Lateral control strategy for autonomous steering of ackerman-like vehicles. Robotics and Autonomous Systems (45),pp. 223-233, 2003.

[6] I. Papadimitriou and M. Tomizuka. Fast lane changing computations using polynomials. Proceedings of the 2003 American Control Conference, pp. 48-53, Denver, June, 2003.

[7] H. Jula, E. B. Kosmatopoulos, and P. A. Ioannou. Collision avoidance analysis for lane changing and merging. IEEE Transactions on Vehicular Technology. vol.49, pp. 2295-2308, 2000.

[8] T. Shamir. Overtaking a slower-moving vehicle by an autonomous vehicle. ICSC Symposium Engineering Intelligent Systems, 2004.

[9] D. Dolgov, S. Thrun, M. Montemerlo, and J. Diebel. Path planning for autonomous vehicles in unknown semi-structured environments. The International Journal of Robotics Research (29), pp. 485-501, 2010.

[10] M. Pivtoraiko and A. Kelly. Efficient constrained path planning via search in state lattices. International Symposium on Artificial Intelligence, Robotics, and Automation in Space, pp. 1-7., 2005.

[11] V. Hundelshausen, F. Himmelsbach, M. Hecker, F. Mueller, A. Wuensche, and H.-J. Wuensche. Driving with tentacles: Integral structures for sensing and motion. Journal of Field Robotics, vol.25, pp.640-673, September 2008.

[12] M. Himmelsbach, T. Luettel, F. Hecker, V. Hundelshausen, and H.-J. Wuensche. Autonomous off-road navigation for mucar-3, improving the tentacles approach: Integral structures for sensing and motion. Kunstl Intell, 2011.

[13] C. Katrakazas, M. Quddus, W.-H. Chen, and L. Deka. Real-time motion planning methods for autonomous on-road driving: State-ofthe-art and future research directions. Transportation Research Part C: Emerging Technologies, 2015.

[14] H. Mouhagir, R. Talj, V. Cherfaoui, F. Aioun, and F. Guillemard. A markov decision process-based approach for trajectory planning with clothoid tentacles. International IEEE Conference on Intelligent Vehicles Symposium (IV), Sweden, June, 2016.

[15] http://www.oktal.fr/fr/automobile/gammes-de-simulateurs/produitslogiciels-scaner.

[16] A. Chebly, G. Tagne, R. Talj, and A. Charara. A local trajectory planning and tracking for autonomous vehicle navigation using clothoid tentacles method. International IEEE Conference on Intelligent Vehicles Symposium (IV) pp. 764-769, Seoul, South Korea, Jun, 2015.

[17] C. Yu, V. Cherfaoui, and P. Bonnifait. An evidential sensor model for velodyne scan grids. 13th International Conference on Control Automation Robotics \& Vision (ICARCV), pp. 583-588, 2014.

[18] T. Gindele, S. Brechtel, and R. Dillmann. Learning driver behavior models from traffic observations for decision making and planning. IEEE Intelligent Transportation Systems Magazine, pp. 69-79, 2015.

[19] https://www.legifrance.gouv.fr/. 